\title{
Improvement of aged lower eyelid by insertion of polydioxanone threads
}

\author{
Ue-Kyoung Hwang, MD, MPH', Yong-Hyun Kwon, $\mathrm{MD}^{2}$, Yong-Jin Cho, $\mathrm{PhD}^{3}$, Kyoung-Jin Kang, MD, $\mathrm{PhD}^{4}$ \\ ${ }^{1}$ Yeline Esthetic Clinic, Bundang, Rep. of Korea, ${ }^{2}$ Bloom Esthetic Clinic, Seoul, Rep. of Korea, ${ }^{3}$ College of Fine Arts, Han-nam University, \\ Daejeon, Korea, ${ }^{4}$ Seoul Cosmetic Surgery Clinic, Busan, Rep. of Korea
}

Orbital fat protrusion, skin laxity with wrinkling, and tear trough deformity with hyperpigmentation of the lower eyelid make individuals appear fatigued and elderly. For cosmetic correction of the aged lower eyelid, either invasive blepharoplasties or less invasive procedures such as filler, high frequency device therapy or laser therapy are popularly performed. Currently in Korea, polydioxanone (PDO) thread is commonly utilized to tighten and lift the aged face. We present a case report demonstrating improvement of orbital fat protrusion, skin laxity with wrinkles, and tear trough deformity following insertion of PDO threads into the lower eyelid of an aging patient. Twenty PDO threads (6.0 USP, $30 \mathrm{~mm}$ in length, New Up PDO suture $\mathrm{Kit}^{\oplus}$ produced by Meditronic Dongjun) were inserted at 1-mm intervals into the submuscular layer and the protruded orbital fat pads of both lower eyelids of a 54-year-old patient. Two months following intervention, the patient presented with flattening of the protruded orbital fat, skin tightening and wrinkle improvement, volume restoration in tear trough deformity, and skin whitening; the patient was satisfied with the results of the therapy. Common complications such as persistent swelling, hematoma, skin irregularity, and infection were not observed during follow-up assessments. Our findings suggest that PDO thread insertion may be a useful, non-invasive procedure to rejuvenate the aged lower eyelid of patients with anxieties regarding potential complications due to invasive lower blepharoplasty.

Keywords: polydioxanone; rejuvenation; aged lower eyelid; orbital fat protrusion' tear trough deformity; lower skin laxity

\section{Introduction}

The aged lower eyelid is typically characterized by skin laxity with wrinkles, protruded orbital fat, and tear trough deformity [1] all potentially caused by aging processes such as bone resorption, attenuation of the retaining ligament and septum, decreases in collagen deposition in the skin and subcutaneous layer, and suborbicularis oculi fat (SOOF) descent $[2,3]$.

To improve the appearance of the aged lower eyelid, invasive blepharoplasty and non-invasive procedures such as hyaluronic acid filler, laser treatments, and high frequency device treatments are commonly performed. Currently, therapeutic trends for cosmetic rejuvenation are shifting to favor less invasive procedures.

Interestingly, many Korean plastic and cosmetic physicians have begun to utilize PDO threads, a suture material employed in open-heart surgeries, for non-invasive cosmetic procedures such as facial tightening and lifting [4]. Importantly, due to their bio-absorbability in tissue, PDO threads have a lower risk of complications.

Moreover, recent reports indicate that insertion of PDO threads into fat tissue induced collagen formation and increased cellular activation by converting mechanical stimulation to cellular signaling (i.e. mechanotransduction) [5]. 
Here, we aimed to improve protruded orbital fat, tear trough deformities, and skin laxity and wrinkling through PDO thread insertion into the aged lower eyelid. Following treatment with this novel technique, we report a notable clinical case demonstrating significant aesthetic improvements in the aged lower eyelid.

\section{Methods}

\section{Patient selection}

A 54-year-old female patient, having not received any eye procedures in the previous 5 years, volunteered for the treatment protocol.

\section{PDO thread device}

A 30-mm length of PDO thread (6.0 USP monofilament, New Up PDO suture $\mathrm{Kit}^{\circledR}$ produced by Meditronic Dongjun Co, Ltd, Seoul, Korea) was equipped to the needle (30 gauge; $25 \mathrm{~mm}$ in length), with $15 \mathrm{~mm}$ located inside the needle and $15 \mathrm{~mm}$ placed on the needle surface (Fig. 1).

\section{PDO thread insertion protocol}

Local anesthesia using a $2 \%$ lidocaine solution with epinephrine $(1: 100,000)$ was administered to the entry sites and pro-

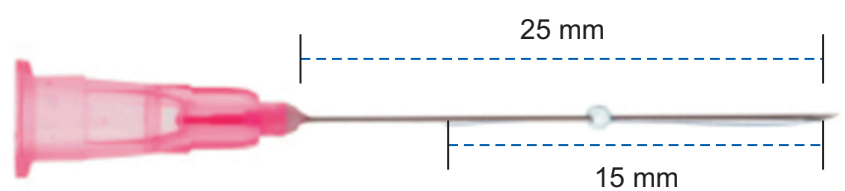

Fig. 1. Schematic figure of the PDO thread device (New Up PDO suture Kit ${ }^{\bullet}$ produced by Meditronic Dongjun Co, Ltd, Seoul, Korea). A $30-\mathrm{mm}$ length of PDO thread (6.0 USP monofilament) was equipped to the needle (30 gauge; $25 \mathrm{~mm}$ in length), with $15 \mathrm{~mm}$ located inside the needle and $15 \mathrm{~mm}$ placed on the needle surface.

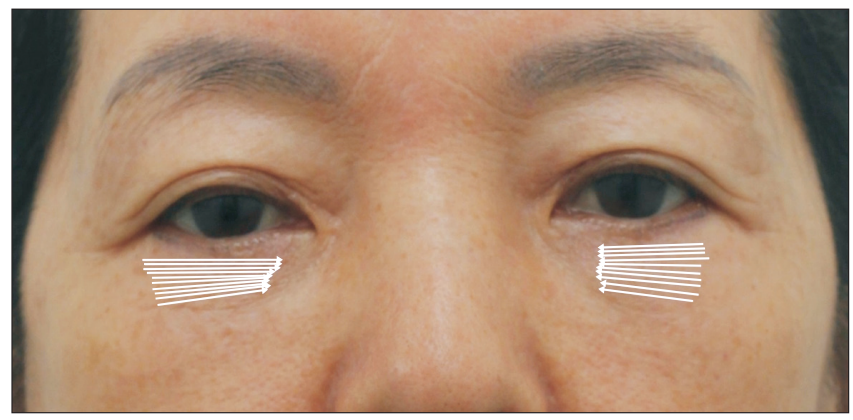

Fig. 2. Schematic view of insertion points for the PDO threads. The threads were inserted into the submuscular layer, protruded orbital fat pad, and ORL (orbital retaining ligament). Gray arrows indicate the direction and placement of the PDO threads. truded orbital fat pad. Ten PDO threads were advanced through the submuscular layer and inserted into the protruded orbital fat pad and orbital retaining ligament at $1 \mathrm{~mm}$ intervals into the lower eye area of the patient as depicted in Fig. 2. During thread insertion, the upper eyelid was maximally opened upward to keep the orbital fat pad prominently displayed. Following thread insertion, the lower eyelid was compressed with gauze and $3 \mathrm{M}$ taping was applied for one day to prevent swelling and further bleeding.

\section{Results}

Eight weeks following insertion of the PDO threads into the aged lower eyelid of the patient, we observed several significant cosmetic improvements (Fig. 3). Specifically, orbital fat protrusion in the right eyelid was significantly decreased and the eyelid appeared flattened and taut. Further, both the width and depth of the tear trough were decreased and the lid/cheek junction appeared more defined. Additionally, eyelid wrinkling was drastically reduced, most notably in the right eyelid, with significant skin whitening and improved tightness.

\section{Discussion}

Orbital fat protrusion, skin laxity with wrinkles, tear trough deformity, and hyper-pigmentation are all recognized charac-
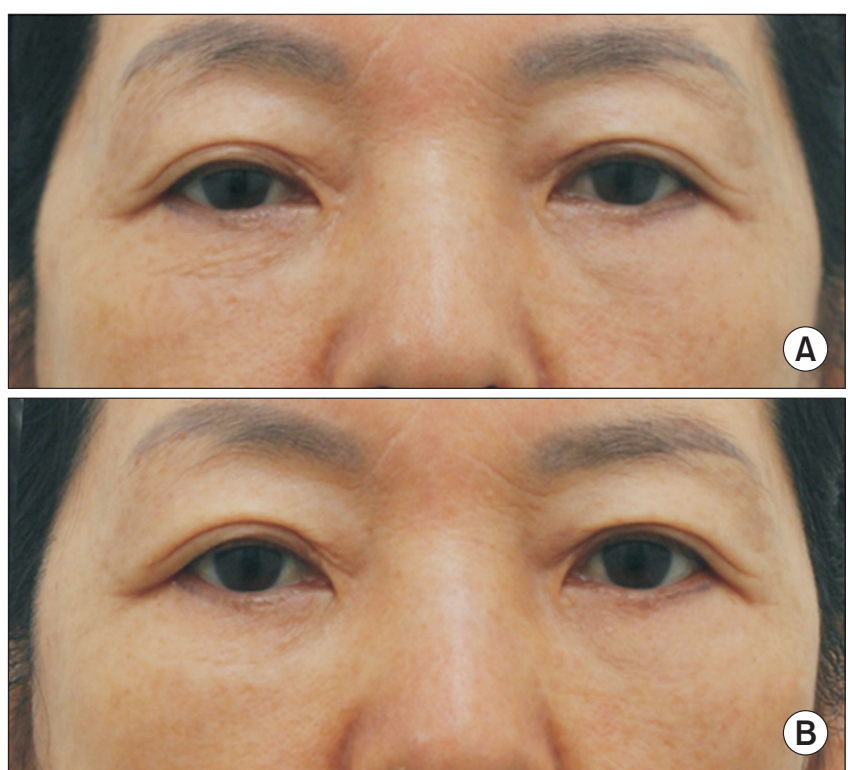

Fig. 3. PDO threads were inserted into the aged lower eyelids of a 54-year-old woman. Significant skin rejuvenation effects were witnessed 8 weeks following treatment. Both the pre-operative (A) and post-operative views (B) are depicted. 
teristics of the aged lower eyelid. As a result, various aesthetic procedures have been developed to improve the appearance of the aged lower eyelid.

Currently, PDO threading is commonly utilized for skin tightening and lifting. As an absorbable suture material, PDO may serve as a tissue scaffold [6]. Mechanistically, this scaffold acts as an activator of intercellular interactions, collagen deposition, neovascularization, and mechanotransduction [5]. Interestingly, mechanotransductive signaling in fibroblasts produced cytoskeletal remodeling following acupuncture of the subcutaneous fat layer [7]. Similarly, previous investigation demonstrated fibrotic encapsulation of PDO threads following insertion into the fat layer. Further, this report suggested that thread encapsulation was caused by infiltration of fibroblasts, potentially leading to contraction of the subcutaneous fat layer [8].

In the current investigation, we endeavored to rejuvenate the appearance of the aged lower eyelid by inserting PDO threads into the tissue. Interestingly, we observed improvements in orbital fat protrusion, likely due to contraction of the fat layer accompanied by fibrotic band formation in the subcutaneous fat layer $[7,8]$.

The improvements in the tear trough were caused by decreases in orbital fat protrusion, tightening and thickening of the orbital retaining ligament, and decreases in skin laxity. As a result, eyelid/cheek delineation became more pronounced. Further, the disappearance of eyelid wrinkling following PDO stimulation was likely attributable to increased collagen synthesis and activation of cell-cell interactions.

Biomechanical stimulation following PDO biodegradation in tissue further caused enhanced whitening of the skin color likely caused by both neovascularization and increased cellular TGF- $\beta$ signaling [9], demonstrated to be an important inhibitor of melanogenesis [10].

Although we report only a single clinical case, our findings suggest that application of PDO thread insertion to the aged lower eyelid may be an effective, alternative method for improving signs of lower eyelid aging. However, additional investigation of treated patients in combination with long term observation will be necessary to demonstrate the safety of this treatment methodology.

\section{Conflicts of interest}

The authors have nothing to disclose.

\section{References}

1. Farkas JP, Pessa JE, Hubbard B, Rohrich RJ. The science and theory behind facial aging. Plast Reconstr Surg Glob Open 2013;1:e8-e15.

2. Levesque AY, de la Torre JI. Midface anatomy, aging, and aesthetic analysis. Facial Plast Surg Clin North Am 2015;23:12936.

3. Friedmann DP, Goldman MP. Dark circles: etiology and management options. Clin Plast Surg 2015;42:33-50.

4. Suh DH, Jang HW, Lee SJ, Lee WS, Ryu HJ. Outcomes of polydioxanone knotless thread lifting for facial rejuvenation. Dermatol Surg 2015;41:720-5.

5. Yoon JH, Kim SS, Kim DI. Skin rejuvenation to make use of absorbable PDO suture in regenerative medicine. Seoul: MD WORLD Medical Publishing Co.; 2012.

6. Moshiri A, Oryan A, Meimandi-Parizi A. Synthesis, development, characterization and effectiveness of bovine pure platelet gel-collagen-polydioxanone bioactive graft on tendon healing. J Cell Mol Med 2015;19:1308-32.

7. Langevin HM, Bouffard NA, Badger GJ, Churchill DL, Howe AK. Subcutaneous tissue fibroblast cytoskeletal remodeling induced by acupuncture: evidence for a mechanotrasduction-based mechanism. J Cell Physiol 2006;207:767-74.

8. Gerarduzzi C, Di Battista JA. Myofibroblast repair mechanisms post-inflammatory response: a fibrotic perspective. Inflamm Res 2017;66:451-65.

9. Kim J, Zheng Z, Kim H, Nam KA, Chung KY. Investigation on the cutaneous change induced by face-lifting monodirectional barbed polydioxanone thread. Dermatol Surg 2017;43:7480.

10. Kim WS, Park SH, Ahn SJ, Kim HK, Park JS, Lee GY, et al. Whitening effect of adipose-derived stem cells: a critical role of TGF-beta 1. Biol Pharm Bull 2008;31:606-10. 